\title{
Existence of a twist angle intrinsic to the reference orbit in circular accelerators
}

\author{
Stefania Petracca* \\ University of Sannio, Benevento, and INFN Salerno, Italy \\ Kohji Hirata* \\ Sokendai, Graduate University for Advanced Studies, Hayama, Japan \\ (Received 31 October 2000; published 27 December 2000)
}

\begin{abstract}
In a circular accelerator, a horizontal-vertical coupling can occur as a purely geometrical effect, in the absence of any coupling device (skew-quadrupole magnets, etc.). The coupling between horizontal and vertical coordinates (and momenta) is represented by a rotation $[\mathrm{SO}(2)]$. The rotation angle is called the twist angle. It comes from the geometry of the reference orbit, and is related to the possible nonintegrability of parallel transport on it. We give an analytic expression of the twist and discuss some simple dynamical consequences of the twist on betatron tune.
\end{abstract}

PACS numbers: 29.20.-c, 29.27.-a

\section{INTRODUCTION}

In particle accelerators, the betatron coordinates are defined with respect to the reference orbit. The reference orbit closes after one turn, but the betatron coordinates do not necessarily come back to the original values after one turn: the horizontal $(x)$ and vertical $(y)$ axes can be rotated by an angle $\Phi$ (twist angle), which is unique and intrinsic to a given configuration of the reference orbit. This effect, related to the anholonomy (nonintegrability) of the (local) coordinate systems set up along nonplanar closed (Jordan) curves, has been well known since Gauss. Its relevance in physics has been stressed by several authors (e.g., Pancharatnam, Berry, and Hannay; see [1]) and is related to the fact that all physical quantities obey the (tensor) parallel transport law. Its possible relevance in connection with accelerators has been recently pointed out $[2,3]$. In this paper, we will discuss its (linear) effect on the betatron oscillations. Preliminary results were given in Refs. [4,5].

The paper is organized as follows. In Sec. II, we define the twist angle; in Sec. III, we give a formula for computing the twist angle; in Sec. IV, we study one possible dynamical consequence. Conclusions follow under Sec. V. In the Appendix we rephrase the concept in terms of the Frenet-Serret (FS) triad.

Throughout the paper we assume, for simplicity, that the reference orbit is composed of smoothly joining planar circular arcs (corresponding to bending magnets) and straight segments.

\footnotetext{
*Also at High Energy Research Organization (KEK), Tsukuba, Japan.
}

\section{A GLOBAL COORDINATE SYSTEM BASED ON PARALLEL TRANSPORT}

Let the reference orbit be described by $\mathbf{r}(s)$, where $s$ is the arc length. Let $\hat{\mathbf{e}}_{z}(s)=\dot{\mathbf{r}}(s) /|\dot{\mathbf{r}}(s)|$ be the unit tangent vector, and

$$
\boldsymbol{\Omega}=\hat{\mathbf{e}}_{z} \times \dot{\hat{\mathbf{e}}}_{z} .
$$

At some point in the ring, e.g., $s=0$, we define two (unit) vectors $\hat{\mathbf{e}}_{x}(0)$ and $\hat{\mathbf{e}}_{y}(0)$ perpendicular to $\hat{\mathbf{e}}_{z}(0)$ such that $\left(\hat{\mathbf{e}}_{x}(0), \hat{\mathbf{e}}_{y}(0), \hat{\mathbf{e}}_{z}(0)\right)$ form a right-handed orthonormal basis. We can then parallel transport $\hat{\mathbf{e}}_{x}(s)$ and $\hat{\mathbf{e}}_{y}(s)$ along the reference orbit. The parallel transport equations $\operatorname{are}^{1}$ [6]

$$
\dot{\hat{\mathbf{e}}}_{i}=\mathbf{\Omega} \times \hat{\mathbf{e}}_{i}, \quad i=x, y, z,
$$

with initial conditions at $s=0$. We can introduce the (local, principal) curvature radii $\rho_{x}$ and $\rho_{y}$ in $\boldsymbol{\Omega}=$ $-\hat{\mathbf{e}}_{y} / \rho_{x}+\hat{\mathbf{e}}_{x} / \rho_{y}$, to put Eq. (2.2) in the form

$$
\left(\begin{array}{c}
\dot{\hat{\mathbf{e}}}_{x} \\
\dot{\hat{\mathbf{e}}}_{y} \\
\dot{\hat{\mathbf{e}}}_{z}
\end{array}\right)=\left(\begin{array}{ccc}
0 & 0 & \rho_{x}^{-1} \\
0 & 0 & \rho_{y}^{-1} \\
-\rho_{x}^{-1} & -\rho_{y}^{-1} & 0
\end{array}\right)\left(\begin{array}{c}
\hat{\mathbf{e}}_{x} \\
\hat{\mathbf{e}}_{y} \\
\hat{\mathbf{e}}_{z}
\end{array}\right) .
$$

Integration of (2.3) gives

$$
\left(\hat{\mathbf{e}}_{x}(s), \hat{\mathbf{e}}_{y}(s), \hat{\mathbf{e}}_{z}(s)\right)^{t}=F(s, 0)\left(\hat{\mathbf{e}}_{x}(0), \hat{\mathbf{e}}_{y}(0), \hat{\mathbf{e}}_{z}(0)\right)^{t},
$$

where

$$
F(s, 0)=(T) \int_{0}^{s} \exp A\left[\rho_{x}(s), \rho_{y}(s)\right] d s,
$$

where $(T)$ stands for the time ordered product and $A$ is the matrix in Eq. (2.3). Equation (2.4) defines the coordinate system for all $s \in[0, C]$, where $C$ is the ring

\footnotetext{
${ }^{1}$ Under parallel transport all three (unit) vectors rotate by the same angle $|\vec{\Omega}| d s$ around the axis $\vec{\Omega} /|\vec{\Omega}|$, after moving from $s$ to $s+d s$ along the orbit.
} 


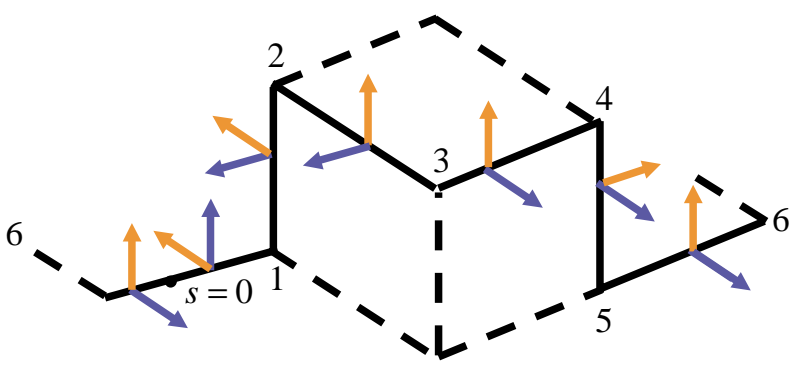

FIG. 1. (Color) The parallel transport and the twist. The point $s=0$ is indicated by a full circle. The magnets (arcs) are numbered. Magnet \#6 corresponds to a long planar arc. The unit vectors $\hat{\mathbf{e}}_{x}$ (dotted arrow) and $\hat{\mathbf{e}}_{y}$ (solid arrow) are defined arbitrarily at $s=0+$, and parallel transported along the reference orbit shown. As they get back to $s=0-$ after a complete turn, they are tilted by an angle $\Phi=\pi / 2$ in the example.

circumference. Since the orbit should be smooth, we have $\hat{\mathbf{e}}_{z}(C)=\hat{\mathbf{e}}_{z}(0)$, which implies

$$
F(C, 0)=\left(\begin{array}{ccc}
\cos \Phi & \sin \Phi & 0 \\
-\sin \Phi & \cos \Phi & 0 \\
0 & 0 & 1
\end{array}\right)
$$

Equation (2.6) defines the twist angle $\Phi$. Now, $F(C, 0)$ can be expressed as

$$
F(C, 0)=f_{n} f_{n-1} \cdots f_{2} f_{1},
$$

where $f_{i}$ accounts for the effect of the $i$ th magnet along the reference orbit

$$
f_{i}=\exp \left\{A\left(\rho_{x}^{i}, \rho_{y}^{i}\right) l_{i}\right\},
$$

with $l_{i}$ being the length of the reference orbit through it.

In Fig. 1 we show a pictorial example of parallel transport along a closed orbit and the related twist angle.

\section{THE TWIST ANGLE AS AN EFFECT OF NONINTEGRABILITY}

The existence of the twist angle is related to the wellknown fact that a vector which is parallel transported ${ }^{2}$ along a closed curve laid on a two-dimensional curved (i.e., nonapplicable ${ }^{3}$ ) surface acquires a net rotation (while preserving its length) with respect to its initial orientation. Conversely, in the simplest case of a planar circular trajectory, where $\rho_{x}=R$ and $\rho_{y}=\infty$, from Eqs. (2.7) and (2.8) we get $\cos (\Phi)=1$, and the twist angle is 0 .

The simplest example of a 2D surface which cannot be applied onto a plane is a sphere. The function components of $\hat{\mathbf{e}}_{z}(s)$ are the coordinates of a point on the unit sphere, and this defines a map from the reference orbit to a unit-

\footnotetext{
${ }^{2}$ The scalar product $(\vec{a}, \vec{b})$ between vectors, and hence the vector length $(\vec{a}, \vec{a})$, are preserved [7] upon parallel transport of $\vec{a}, \vec{b}$, and so is the angle $(\vec{a}, \vec{b}) / \sqrt{(\vec{a}, \vec{a})(\vec{b}, \vec{b})}$.

${ }^{3}$ Applicable surfaces can be deformed continuously into a plane, without cuts or overlaps.
}

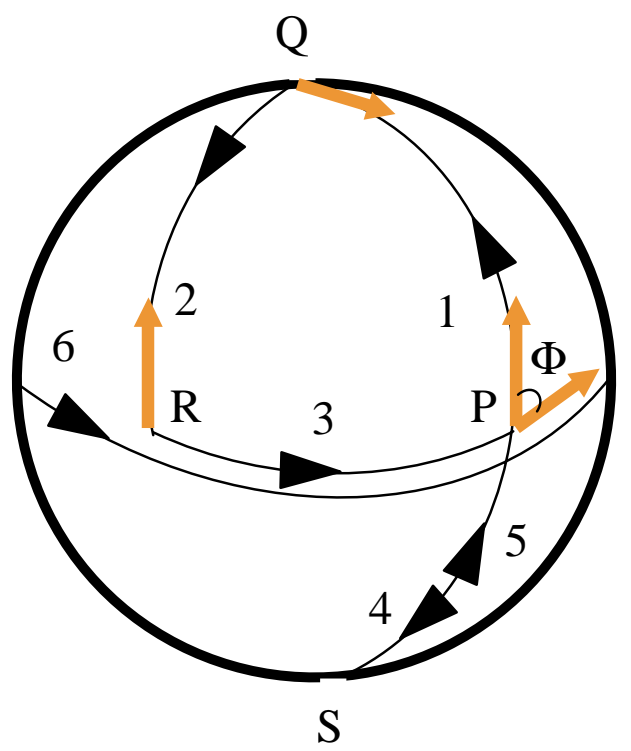

FIG. 2. (Color) The geodesic curve $\Gamma$ described by $\hat{\mathbf{e}}_{z}$ on the unit-radius sphere $S^{2}$ corresponding to Fig. 1. The unit vector $\hat{\mathbf{e}}_{x}$ is displayed at each point on $\Gamma$. At $s=0+, \hat{\mathbf{e}}_{z}$ is at $\mathrm{P}$ in the figure, and $\hat{\mathbf{e}}_{x}$ (the dotted arrow) lies along the equator. Entering magnet \#1, $\hat{\mathbf{e}}_{z}$ moves to $\mathrm{Q}$, and $\hat{\mathbf{e}}_{x}$ is parallel transported. The same happens moving through magnets \#2 and \#3. Then $\hat{\mathbf{e}}_{z}$ comes back to $P$. In going through magnets \#4 and \#5 from $\mathrm{P}$ to $\mathrm{S}, \hat{\mathbf{e}}_{z}$ makes a round-trip (null contribution to $\Phi$ ). The same happens going through magnet \#6, along the equator $(2 \pi$ contribution to $\Phi)$. On returning to $\mathrm{P}$, $\hat{\mathbf{e}}_{x}$ is tilted by $\Phi=\pi / 2$. The solid angle is $\pi / 2+2 \pi[\equiv \Phi, \bmod (2 \pi)]$.

radius spherical surface $S^{2}$, tracing a closed curve $\Gamma$ on it; see Fig. 2, which corresponds to the configuration in Fig. 1. From Eq. (2.1) it is seen that $\Gamma$ is (piecewise) geodesic on $S^{2}$.

At each point on $\Gamma$, we can attach the corresponding unit vector $\hat{\mathbf{e}}_{x}(s)$, lying on its tangent plane. ${ }^{4}$ The twist angle $\Phi$ is then the angle between $\hat{\mathbf{u}}(0+)$ and $\hat{\mathbf{u}}(0-)$, after one turn of the reference orbit.

Let $\phi(s)$ be the angle between $\hat{\mathbf{e}}_{x}(s)$ and the local tangent vector of $\Gamma$. The angle $\phi(s)$ is constant along each geodesic. At each corner on $\Gamma$ (labeled by $\mathrm{Q}, \mathrm{R}$, etc. in Fig. 2), $\phi$ undergoes an abrupt change, $\phi \rightarrow \phi-\alpha_{i}=$ $\phi-\pi+\beta_{i}$, where $\alpha_{i}\left(\beta_{i}\right)$ is the exterior (interior) angle of the corner. After a complete turn, the tangent vector of $\Gamma$ has undergone a $2 \pi$ rotation. Hence ${ }^{5}$

$$
\Phi=2 \pi-\sum_{i} \alpha_{i}=(\text { solid angle subtended by } \Gamma) .
$$

Equation (3.1) is the well-known Gauss-Bonnet theorem [7]. The twist angle reflects the nonintegrability of the

\footnotetext{
${ }^{4}$ The same procedure applies to $\hat{\mathbf{e}}_{y}(s)$.

${ }^{5}$ In Eq. (3.1) the solid angle is oriented, and $\Phi$ is defined as $\bmod (2 \pi)$.
} 
transport of $\hat{\mathbf{e}}_{x}$ along $\Gamma$ and is an example of the holonomy group on $S^{2}$. Note once more that $\Phi$ does not depend on the choice of the coordinate frame. It is an intrinsic, i.e., physical, object. It is easily seen that $\Phi$ does not change when we start from a point different from $s=0$.

An additional rotation $-\Phi$ should therefore be applied somewhere in the ring in order to make the one turn matrix $F(C, 0)$ an identity.

\section{DYNAMICAL EFFECT OF THE TWIST ANGLE}

When a nonzero twist angle $\Phi$ exists, the $(x, y)$ coordinates should be adjusted, e.g., at $s=0$,

$$
\left(\begin{array}{l}
x \\
y
\end{array}\right)_{0+}=\left(\begin{array}{cc}
\cos \Phi & \sin \Phi \\
-\sin \Phi & \cos \Phi
\end{array}\right)\left(\begin{array}{l}
x \\
y
\end{array}\right)_{0-} .
$$

This should be taken into account, no matter what dynamics are concerned, including linear and nonlinear betatron oscillations, polarizations, coherent dynamics, etc. As an example, we focus here on the simplest case of linear betatron oscillations.

We assume that the bending and quadrupole magnets are chosen in such a way that the $\rho$ 's are either horizontal or vertical with respect to $\left(\hat{\mathbf{e}}_{x}(s), \hat{\mathbf{e}}_{y}(s)\right)$, so that the transfer matrix is always piecewise diagonal. The one turn matrix thus acquires the form

$$
M=R \operatorname{diag}\left(m_{x}, m_{y}\right),
$$

where $R$ is the rotation in Eq. (4.1), and the $m$ 's are $2 \times 2$ symplectic matrices.

As an example, let us assume

$$
m_{x, y}=\left(\begin{array}{cc}
\cos \left(2 \pi \nu_{x, y}\right) & \beta_{x, y} \sin \left(2 \pi \nu_{x, y}\right) \\
-\beta_{x, y}^{-1} \sin \left(2 \pi \nu_{x, y}\right) & \cos \left(2 \pi \nu_{x, y}\right)
\end{array}\right)
$$

as a generalization of the Möbius ring [8], which corresponds to $\Phi=\pi / 2$. By standard techniques [9], one can

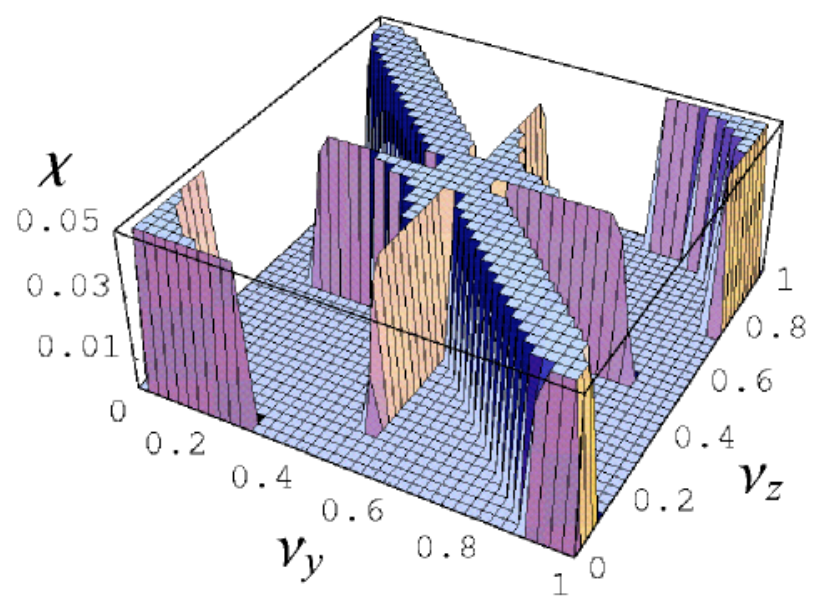

FIG. 3. (Color) The unstable region in the $\left(\nu_{x}, \nu_{y}\right)$ plane, for $\Phi=0.04 \pi, \beta_{x}=0.33 \mathrm{~m}, \beta_{y}=0.01 \mathrm{~m}$. The vertical axis is $\chi \equiv($ growth rate -1$)$. calculate the eigenvalues of $M$. The largest of these is the so-called growth rate, and is an obvious measure of stability.

The unstable region in the $\left(\nu_{x}, \nu_{y}\right)$ plane is shown in Fig. 3, for $\Phi=0.04 \pi, \beta_{x}=0.33 \mathrm{~m}$, and $\beta_{y}=0.01 \mathrm{~m}$. It is seen that, perhaps surprisingly, a small twist angle $\Phi$ produces a large unstable region, as seen from the quantity $\chi \equiv($ growth rate -1$)$

Note that the instability pattern is left unchanged when we make the transformations $\left(\nu_{x}, \nu_{y}\right) \rightarrow\left(\nu_{x}+1 / 2, \nu_{y}+\right.$ $1 / 2)$ or $\Phi \rightarrow \Phi+\pi$.

\section{CONCLUSIONS}

In this paper we pointed out the presence of an intrinsic $\mathrm{SO}(2)$ rotation associated with the configuration of the reference orbit. The twist angle $\Phi$ is unique for a given reference orbit and is a physical object, which should be included in the tracking.

We have discussed the simplest dynamical effect of the twist angle. Further study will clarify its effects on nonlinear dynamics, polarization (spin), coherent instabilities, etc.

\section{ACKNOWLEDGMENTS}

This work was sponsored in part by the European Community through a grant to Stefania Petracca at KEK, Tsukuba, Japan. S.P. wishes to thank the Accelerator Theory Group at KEK for gracious hospitality and stimulating discussions.

\section{APPENDIX: THE FRENET-SERRET TRIAD}

The main point of this paper has been to elucidate that to any nonplanar (simple, smooth) fiducial orbit it is possible to associate in an unique way an angle $\Phi$ of entirely geometric origin. This can be computed in either of the following ways: (1) as the net rotation undergone by any (transverse) vector parallel transported through one complete revolution along the orbit or (2) as the algebraic sum of all jump rotations undergone by the Frenet-Serret (transverse) vectors in passing through the closed orbit inflection and deflection points.

In this Appendix we show how $\Phi$ emerges from the Frenet-Serret triad.

To any curve $\Lambda$ of class $C^{m}(m \geq 2)$ parametrically represented by $\vec{r}=\vec{r}_{0}(s), s$ being the arc length measured from some origin on $\Lambda$, we can attach a local FS triad,

$$
\hat{t}=\frac{d \vec{r}_{0}}{d s}, \quad \hat{n}=\frac{d^{2} \vec{r}_{0}}{d s^{2}}\left|\frac{d^{2} \vec{r}_{0}}{d s^{2}}\right|^{-1}, \quad \hat{b}=\hat{t} \times \hat{n} .
$$

The unit vectors $\vec{n}, \vec{b}, \vec{t}$ have the following meaning: $\vec{t}$ is the local tangent, $\vec{n}$ is the normal oriented toward the center of curvature, and $\vec{b}$ is the binormal; $\vec{n}$ and $\vec{t}$ define the 
local (osculating) curve plane. ${ }^{6}$ The FS does not transport continuously. If the osculating planes of two arcs joining at $P$ are not parallel, it exhibits [3] an abrupt rotation across $P$. Even in smooth curves ( $\hat{t}$ continuous) the transverse vector pair $(\hat{n}, \hat{b})$ may exhibit abrupt discontinuities across inflection and deflection points. Across an inflection point, say at $s=0$ ( $\hat{t}$ being continuous), one has

$$
\hat{n}\left(0^{+}\right)=-\hat{n}\left(0^{-}\right), \quad \hat{b}\left(0^{+}\right)=-\hat{b}\left(0^{-}\right),
$$

whereas across a deflection point, where two arcs join smoothly while their osculating planes make an angle $\delta$, one has

$$
\left(\begin{array}{l}
\hat{n} \\
\hat{b}
\end{array}\right)_{s=0^{+}}=\left(\begin{array}{cc}
\cos \delta & \sin \delta \\
-\sin \delta & \cos \delta
\end{array}\right)\left(\begin{array}{l}
\hat{n} \\
\hat{b}
\end{array}\right)_{s=0^{-}} .
$$

The FS triad is thus transported along the ring using only the following simple rules: (i) moving the FS triad along a plane (torsion-free) circular arc of radius $R$ and length $s=R \theta$ produces a $\theta$ rotation around the (local) $\hat{b}$ vector, (ii) moving the FS triad across an inflection point produces a $\pi$ rotation along the (local) $\hat{t}$ vector, (iii) moving the FS triad across a deflection point where the osculating plane jumps by an angle $\delta$ produces a rotation given by (A3) along the (local) $\hat{t}$ vector, (iv) moving the FS triad along a straight section does not produce any change. Note that $\hat{t}$ is continuous along the closed orbit and coincides with $\hat{\mathbf{e}}_{z}$.

Summing up all jump rotations of the FS triads along a (simple, smooth) nonplanar closed curve, representing the reference orbit, gives, in general, a result different from 0 $[\bmod (2 \pi)]$.

This is exemplified in Fig. 4, where the orbit features one inflection and five deflections. Letting $\delta_{i}$ be the angle between arcs $i$ and $i+1$, Eq. (A3) should be applied with

\footnotetext{
${ }^{6}$ On a straight line, the FS triad is not unique, so that we define it by parallel transport from the preceding arc.
}

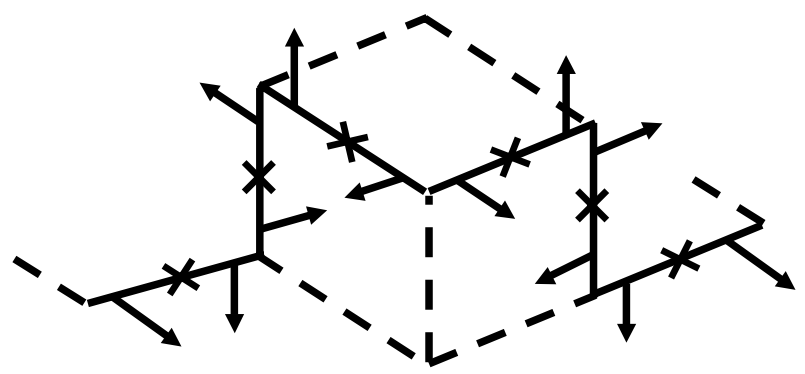

FIG. 4. The Frenet-Serret vector $\hat{n}$ for the same example as in Fig. 1. Arc inflection (deflection) points are highlighted.

$\delta=\delta_{i}$ to describe the jump of the pair $(\hat{n}, \hat{b})$ between $s=s_{i}^{-}$and $s=s_{i}^{+}$. For the special case of Fig. 1, we have

$$
\left(\delta_{1}, \ldots, \delta_{6}\right)=(\pi / 2, \pi / 2,-\pi / 2, \pi,-\pi / 2, \pi / 2) .
$$

It is easily seen that $\delta_{i}$ is the same as the exterior angle $\alpha_{i}$ mentioned in Sec. III.

[1] A. Shapere and F. Wilczek, Geometric Phases in Physics (World Scientific, Singapore,1989).

[2] K. Hirata, in Proceedings of the Second Advanced ICFA Beam Dynamics Workshop, edited by J. Hagel and E. Keil (CERN Report No. 88-04, 1988), p. 62.

[3] E. Forest and K. Hirata, KEK Report No. 92-12, 1992.

[4] S. Petracca and K. Hirata, KEK Report No. 96-52, 1996.

[5] S. Petracca and K. Hirata, in Proceedings of the 5th European Particle Accelerator Conference, Sitges, Spain, 1996, edited by S. Myers et al. (Institute of Physic, Bristol, UK, 1996), p. 959.

[6] K. Yokoya, KEK Report No. 85-7, 1985.

[7] C. Eisenhart, Riemannian Geometry (Cambridge University, Cambridge, England, 1966).

[8] R. Talman, Phys. Rev. Lett. 74, 1590 (1995).

[9] E. D. Courant and H.S. Snyder, Ann. Phys. (Paris) 3, 1 (1958). 